\title{
Building an irreversible Carnot-like heat engine with an overdamped harmonic oscillator
}

\author{
Carlos A. Plata \\ Dipartimento di Fisica e Astronomia "Galileo Galilei", INFN, Università di Padova, \\ Via Marzolo 8, 35131 Padova, Italy \\ David Guéry-Odelin \\ Laboratoire Collisions, Agrégats, Réactivité, IRSAMC, Université de Toulouse, \\ CNRS, UPS, Toulouse, France
}

Emmanuel Trizac

Université Paris-Saclay, CNRS, LPTMS, 91405, Orsay, France.

\begin{abstract}
A. Prados
Física Teórica, Universidad de Sevilla, Apartado de Correos 1065, E-41080 Sevilla, Spain
\end{abstract}

\begin{abstract}
We analyse non-equilibrium Carnot-like cycles built with a colloidal particle in a harmonic trap, which is immersed in a fluid that acts as a heat bath. Our analysis is carried out in the overdamped regime. The cycle comprises four branches: two isothermal processes and two locally adiabatic ones. In the latter, both the temperature of the bath and the stiffness of the harmonic trap vary in time, but in such a way that the average heat vanishes for all times. All branches are swept at a finite rate and, therefore, the corresponding processes are irreversible, not quasi-static. Specifically, we are interested in optimising the heat engine to deliver the maximum power and characterising the corresponding values of the physical parameters. The efficiency at maximum power is shown to be very close to the Curzon-Ahlborn bound over the whole range of the ratio of temperatures of the two thermal baths, pointing to the near optimality of the proposed protocol.
\end{abstract}

\section{Introduction}

The investigation of heat engines is a pillar of classical thermodynamics [1]. The practical interest of the conversion of thermal energy into mechanical work led to unravel the laws of thermodynamics. These laws have been well formulated since the 19th century for macroscopic systems, for which fluctuations are negligible. In this context, the Carnot heat engine has played a major role: the Carnot cycle comprises two isothermal and two adiabatic branches, which are swept in a quasi-static, reversible, 
way. This reversible Carnot heat engine maximises the efficiency but the infinite time operation entails that the delivered power vanishes. In the adiabatic branches, the system is thermally isolated from the bath and there is no heat exchange - moreover, reversibility implies that there is no entropy variation either.

The extension of thermodynamic results to mesoscopic systems, where fluctuations are of paramount importance, is not straightforward ; stochastic thermodynamics has been developed to this end [2 [4], the focus of which lies on non-equilibrium dynamics. In recent years, researchers have looked into the possibility of speeding up the relaxation of physical systems between two given equilibrium states [5-11]. The "engineered swift equilibration" (ESE) techniques that emerged, which have also been referred to as "shortcut to isothermality" are essentially equivalent and can be viewed as the counterpart in the classical realm of the "shortcuts to adiabaticity" (STA) developed in quantum systems [12]. Both STA and ESE processes make it possible to connect given initial and target states in a time that is much shorter than the natural characteristic relaxation time of the system at hand. To avoid confusion, it is perhaps worthwhile pointing that "adiabaticity" in "STA" refers to a slow variation, not to he absence of heat transfer, at variance with terminology to be used below.

STA and ESE techniques make it possible to build heat engines that connect equilibrium states in a finite time, i.e. in an irreversible way. Therefore, the irreversible counterparts of the classical heat engines can be constructed at the mesoscopic level. In fact, our main goal is building an irreversible version of the Carnot heat engine with a colloidal particle in a harmonic trap of stiffness $k$, immersed in a fluid at equilibrium with temperature $T$. This system is relevant from both the theoretical and experimental standpoints. However, a difficulty arises: for mesoscopic systems, it is impossible to completely decouple the system from the heat bath to thermally isolate it - the interaction between a Brownian particle and the fluid in which it is immersed cannot be switched off. Moreover, zero heat and no entropy increment are not equivalent for finite time processes.

The above discussion entails that the definition of adiabatic - in the thermodynamical sense - process is far from trivial at the mesoscale. Notwithstanding, very recently, finite-time adiabatic processes have been characterised for a wide class of mesoscopic systems [13], in the overdamped description of the dynamics. In these processes, the average heat vanishes for all times but there is entropy creation, as imposed by the second principle. We employ these finite-time adiabatic processes to build the corresponding adiabatic branches of the irreversible Carnot engine. Therefore, our approach differs from other recent attempts to construct an irreversible Carnot engine [14 18], the limitations of which are discussed in what follows. Specifically, we focus on the respective definitions of "adiabaticity". In Ref. [14], working in the overdamped regime, the term adiabatic has been employed for a process in which the bath temperature $T$ is instantaneously changed, while the configurational distribution is frozen. However, as already noted by the authors of that work, neither heat nor the entropy increment vanishes in such a process, which are thus non-adiabatic, because of the kinetic contri- 
bution thereto. In Refs. [15, 17, 18, the adiabatic branches are constructed by changing both the temperature of the bath and the stiffness $k$ of the trap but keeping the ratio $T^{2} / k$ constant, which is obtained in the underdamped description. Nevertheless, the condition $T^{2} / k=$ const. has been shown to correspond to isoentropic processes only in the quasi-static limit 15, 19, so such a process is not adiabatic either for finite time operation. $\ddagger$ A completely different approach is proposed in Ref. [16. Therein, the oscillator follows a Hamiltonian dynamics and is completely decoupled from the heat bath during the adiabatic branches, a procedure that cannot be implemented with a Brownian particle immersed in a fluid.

The performance of a heat engine is characterised by its efficiency and power. The maximum efficiency achievable operating between a hot bath at temperature $T_{h}$ and a cold bath at temperature $T_{c}$ is the well-known Carnot efficiency $\eta_{\mathrm{C}}=1-T_{c} / T_{h}$. However, it is only reached for infinite time operation, which makes the power vanish. The cycle must be swept in a finite time to yield a nonzero power output. This acceleration of the process entails a non-equilibrium dynamics and reduces the reachable efficiency. The study of efficiency at maximum power is a classical problem associated with the field of finite-time thermodynamics 20 25]. Curzon and Ahlborn derived that the efficiency at maximum power for a macroscopically endoreversible heat engine is given by $\eta_{\mathrm{CA}}=1-\sqrt{T_{c} / T_{h}}[20$.

There is no general proof ensuring that the efficiency of any arbitrary heat engine at maximum power is bounded by the Curzon-Ahlborn value. Nevertheless, myriads of different studies hint at the existence of some universal properties, connected to the Curzon-Ahlborn bound, for the efficiency at maximum power. Specifically, it has been proven that in the limit of small relative temperature difference, the two first terms in the expansion of the efficiency at maximum power in the Carnot efficiency are universal 26 28. This finding is completely consistent with the results for the efficiency at maximum power in different stochastic heat engines constructed either with a Brownian particle [14], a Feynman ratchet [29], or a quantum dot [30].

Another main objective of our work is the optimisation of the irreversible Carnot engine. Specifically, in connection with the discussion above, we are interested in looking into the optimisation in a sense to be specified soon below, of the delivered power and its associated efficiency. In this regard, the optimal protocols for isothermal and the adiabatic branches, which have been explicitly worked out recently $13,14,31,32$, play a crucial role. It appears that work should be minimised in the isothermal processes 14,31,32, whereas the connection time is minimised in the adiabatic ones [13].

The rest of the paper is organised as follows. In section 2, we introduce the model system with which we construct our heat engine: a Brownian particle moving in a harmonic trap. Special attention is paid to its energetics. Section 3 is devoted to putting forward the optimal protocols for both the isothermal and adiabatic branches.

$\ddagger$ Reference 13], although working in the overdamped description, incorporates the kinetic contribution to the energy balance. Therein, the ratio $T^{2} / k$ has been shown to be a non-decreasing function of time for finite-time adiabatic processes, being constant only in the quasi-static limit. 
These protocols allow us to build the Carnot-like cycle, which is analysed in section 4. The efficiency at maximum power is thoroughly investigated in section 5. In section 6 . the main conclusions of our work are presented. Finally, we refer to the Supplementary Material for some further technical details, which complement the main text.

\section{The model system}

\subsection{Definition}

We consider a one-dimensional (1D) overdamped harmonic oscillator of stiffness $k$ in contact with a thermal bath at temperature T. A Brownian particle, confined by optical tweezers, provides an accurate realisation. The stochastic dynamics of the system may be modelled at either the Langevin or the Fokker-Planck levels of description. The average variance of the oscillator $\left\langle x^{2}\right\rangle$, which is given by

$$
\lambda \frac{d\left\langle x^{2}\right\rangle}{d t}=-2 k\left\langle x^{2}\right\rangle+2 k_{B} T
$$

being $k_{B}$ the Boltzmann constant and $\lambda$ the friction coefficient. Physically, we are considering that the harmonic oscillator is immersed in a certain fluid that plays the role of the heat bath, which provides the values of the temperature $T$ and the friction coefficient $\lambda$. Throughout our work, we take $\lambda$ as constant $\S$, time-independent, but we assume both the stiffness of the oscillator $k$ and the bath temperature $T$ to be externally controlled. While the time control of trap stiffness is now routinely achieved experimentally, we refer to Refs. [4,33] for the time control of temperature.

At any time $t$, the state of the system is characterised by the state-point $\left(k,\left\langle x^{2}\right\rangle, T\right)$. Equilibrium states fulfil the equation of state $\left\langle x^{2}\right\rangle_{\mathrm{eq}}=k_{B} T / k$. The above relation, which has been obtained by making the rhs of equation (1) vanish, defines the equilibrium surface in the $\left(k,\left\langle x^{2}\right\rangle, T\right)$ three-dimensional space. We want to describe the energetics of this system at the average level. Thus we define the average energy

$$
E=\frac{1}{2} k\left\langle x^{2}\right\rangle+\frac{1}{2} k_{B} T,
$$

where we have taken into account that the velocity variable is always at equilibrium in the overdamped limit. The equilibrium value of the energy is then $E_{\text {eq }}=k_{B} T$.

Let us now consider a process starting from a certain state $A$ and ending in another state $B$. Work and heat are defined by the relations [2]

$$
\begin{aligned}
& W_{A B}=\frac{1}{2} \int_{A}^{B}\left\langle x^{2}\right\rangle d k, \\
& Q_{A B}=\frac{1}{2} \int_{A}^{B}\left(k d\left\langle x^{2}\right\rangle+k_{B} d T\right)=\frac{1}{2} \int_{A}^{B} k d\left\langle x^{2}\right\rangle+\frac{k_{B}}{2}\left(T_{B}-T_{A}\right),
\end{aligned}
$$

where $T_{A}$ and $T_{B}$ are the temperature values for the initial and final states, $A$ and $B$, respectively. Thus, the first law of thermodynamics reads $\Delta E \equiv E_{B}-E_{A}=W_{A B}+Q_{A B}$.

$\S$ Indeed, when considering a colloidal particle in an optical trap, $\lambda$ is rooted in the solvent viscosity and is essentially constant. 
We have used the following sign convention: for $W, Q>0$ energy is transferred from the environment to the system, whereas for $W, Q<0$ energy is transferred from the system to the environment, irrespective of the "kind" of energy involved. Therefore, in order to consider a heat engine, we are interested in cycles with a negative total work.

\subsection{Non-dimensional variables}

First of all, we introduce dimensionless variables as follows: we divide the stiffness and the temperature by their respective initial values, $\kappa=k / k_{0}, \theta=T / T_{0}$, and the variance by its equilibrium value at the initial temperature, $y=\left\langle x^{2}\right\rangle /\left\langle x^{2}\right\rangle_{\text {eq }, 0}=k_{0}\left\langle x^{2}\right\rangle /\left(k_{B} T_{0}\right)$. Then, we have that $y(t=0)=1$ if the system starts from an equilibrium state. Second, a dimensionless time is defined as $s=k_{0} t / \lambda$. With the above definitions, the evolution of the system in non-dimensional variables is governed by

$$
\frac{d y}{d s}=-2 \kappa y+2 \theta
$$

where the equilibrium surface (or equation of state) reads,

$$
\kappa y_{\mathrm{eq}}=\theta \text {. }
$$

Regarding the energetics, we introduce the dimensionless energy by dividing $E$ by the equilibrium value at the initial time, $k_{B} T_{0}$. Consistently, non-dimensional work and heat are defined with the same energy unit, that is,

$$
\begin{aligned}
& \mathcal{E}=\frac{1}{2} \kappa y+\frac{1}{2} \theta \\
& \mathcal{W}_{A B}=\frac{1}{2} \int_{A}^{B} y d \kappa, \quad \mathcal{Q}_{A B}=\frac{1}{2} \int_{A}^{B} \kappa d y+\frac{1}{2}\left(\theta_{B}-\theta_{A}\right) .
\end{aligned}
$$

The first law reads $\Delta \mathcal{E} \equiv \mathcal{E}_{B}-\mathcal{E}_{A}=\mathcal{W}_{A B}+\mathcal{Q}_{A B}$, and the equilibrium value of the energy is $\mathcal{E}_{\text {eq }}=\theta$.

In dimensionless variables, the state of the system is characterised by the statepoint $(\kappa, y, \theta)$ at any time $s$. For our purposes, it is useful to consider the movement of the projection of the state-point onto the $(\kappa, y)$ plane. In particular, the work $\mathcal{W}$, as given by equation (8), is proportional to the area below the curve $(\kappa(s), y(s))$ swept by the system as time increases.

\section{Building blocks of the cycle: Isothermal and adiabatic processes}

Herein, we aim at building an irreversible heat engine with the above described overdamped harmonic oscillator. Our heat engine operates cyclically between a "hot" source, at dimensionless temperature $\theta_{h}$, and a "cold" source, at temperature $\theta_{c}<\theta_{h}$. Specifically, the non-equilibrium cycle comprises four different processes: two isothermal ones, at temperatures $\theta_{h}$ and $\theta_{c}$, and two locally adiabatic ones that connect the isotherms. No heat is exchanged in average during these locally adiabatic processes at all times, as described below. This is the usual use of the term adiabatic in equilibrium 
thermodynamics, in which adiabatic is employed for a process in which the system is thermally insulated from the environment.

In each cycle, the engine takes energy from the hot reservoir as heat, $\mathcal{Q}_{h}>0$, and performs work, that is, $\mathcal{W}<0$. Therefore, the projection of the state-point onto the $(\kappa, y)$ plane sweeps a certain closed curve $(\kappa(s), y(s))$, which characterises the considered cycle, in the counterclockwise direction. In the light of the above, isothermal and adiabatic processes can be considered as the building blocks for our irreversible heat engine. In the following, we summarise some results obtained in previous studies for isothermal 14, 32] and adiabatic processes [13].

\subsection{Isothermal processes}

We consider two kinds of isothermal processes at temperature $\theta$ : quasi-static and optimal. In both of them, the initial and final states characterised by $\left(\kappa_{A}, y_{B}\right)$ and $\left(\kappa_{A}, y_{B}\right)$, respectively, correspond to equilibrium situations. Therefore, $\kappa_{A} y_{A}=\kappa_{B} y_{B}=$ $\theta$.

First, we deal with the quasi-static case. Therein, $\kappa$ is slowly tuned in such a way that the system sweeps the equilibrium curve $y(s)=\theta / \kappa(s)$ in the $(\kappa, y)$ plane. Therefrom,

$$
\mathcal{W}=\frac{\theta}{2} \ln \frac{\kappa_{B}}{\kappa_{A}}, \quad \mathcal{Q}=-\mathcal{W}, \quad \Delta E=0, \quad \mathcal{E}_{B}=\mathcal{E}_{A}=\theta
$$

Of course, this quasi-static process takes an infinite time.

Second, we look into the optimal process for a given finite time $s_{\mathrm{f}}$. Therein, we are interested in the process for which the work performed by an external agent on the system is minimum, or in other words, we look for the maximum work produced by the system. The evolution of the variance in the optimal process is 14,32 .

$$
\tilde{y}(s)=\left[\sqrt{y_{A}}+\left(\sqrt{y_{B}}-\sqrt{y_{A}}\right) \frac{s}{s_{\mathrm{f}}}\right]^{2} .
$$

From now, tilde denotes optimality in some sense: either for the profiles or for the values of the physical quantities or parameters. Note that $\tilde{y}(s)$ is continuous in the whole interval $\left[0, s_{\mathrm{f}}\right]$.

The optimal evolution for the stiffness is obtained from the evolution equation (5) in the open interval $\left(0, s_{\mathrm{f}}\right)$,

$$
\tilde{\kappa}(s)=\frac{\theta}{\tilde{y}(s)}-\frac{1}{2} \frac{d}{d s} \ln \tilde{y}(s), \quad 0<s<s_{f} .
$$

We recall that the stiffness is discontinuous at both the initial and final times, $\tilde{\kappa}(s=$ $0)=\kappa_{A}, \tilde{\kappa}\left(s=s_{\mathrm{f}}\right)=\kappa_{B}$. In this problem, the elastic constant $\kappa(s)$ plays the role of the "control" function in optimal control theory 34, 35. Similar discontinuities in the "control" function have been repeatedly found in stochastic thermodynamics 6, $14,31,32,36,38, \ldots$

|| This is a consequence of the corresponding "Lagrangian" being linear in the "velocities" 39], which is sometimes called the Miele problem [40]. 
The optimal values of work and heat can also be readily calculated. The results are

$$
\widetilde{\mathcal{W}}=\frac{\theta}{2} \ln \frac{\kappa_{B}}{\kappa_{A}}+\frac{\theta}{s_{\mathrm{f}}}\left(\frac{1}{\sqrt{\kappa_{B}}}-\frac{1}{\sqrt{\kappa_{A}}}\right)^{2}, \quad \widetilde{\mathcal{Q}}=-\widetilde{\mathcal{W}},
$$

Of course, in this isothermal process there is no energy change between the initial and final states $\Delta \mathcal{E}=0, \quad \mathcal{E}_{B}=\mathcal{E}_{A}=\theta$. Note, however, that the energy of the system does change in the intermediate times, $\mathcal{E}(s) \neq \theta$ for $0<s<s_{\mathrm{f}}$ because we are dealing with a non-equilibrium process and $y(s) \neq \theta / \kappa(s)$, as expressed by equation (11).

\subsection{Adiabatic processes}

Now we turn our attention to adiabatic processes, there is no heat transfer at any point of the system trajectory. Therefore, bearing in mind equation (8) we have that the infinitesimal heat vanishes, i.e.

$$
đ Q \equiv \kappa d y+d \theta=0,
$$

Note that temperature becomes a function of time that goes from $\theta_{A}$ to $\theta_{B}$ in adiabatic processes. Similarly to the case of isothermal processes, we only consider adiabatic processes connecting two equilibrium states and then $\kappa_{A} y_{A}=\theta_{A}, \kappa_{B} y_{B}=\theta_{B}$.

The energetics of adiabatic processes is quite simple. The energy change is given by the change in temperature, $\mathcal{E}_{A}=\theta_{A}, \mathcal{E}_{B}=\theta_{B}, \Delta \mathcal{E}=\theta_{B}-\theta_{A}$. Since there is no heat exchange, $\mathcal{Q}=0$, work coincides with the energy change, $\mathcal{W}=\theta_{B}-\theta_{A}$. The above expressions for energy, heat and work apply for any adiabatic process, regardless of its duration, and therefore are valid for both quasi-static and non-equilibrium processes. Nevertheless, the equivalence between adiabatic and isoentropic processes occurs only in the quasi-static limit. It is in the non-equilibrium case that we deviate from the proposals in Refs. [15,17].

Again we consider two kinds of processes: quasi-static and optimal. First, in the quasi-static case, $\kappa$ and $\theta$ are tuned in an infinitely slow way to allow the system sweep the equilibrium curve (6). Combining equations (6) and (13), one gets

$$
y(s)=\frac{y_{A} \theta_{A}}{\theta(s)}=y_{A} \sqrt{\frac{\kappa_{A}}{\kappa(s)}} .
$$

Second, we investigate optimal adiabatic processes. Here, optimal means something different from the sense we used in the previous section. As already said above, the work value is fixed by the initial and target states and thus cannot be optimised. However, two arbitrary states cannot be connected by an adiabatic transformation, the following inequality

$$
\frac{\theta(s)}{\theta_{A}} \geq\left(\frac{y(s)}{y_{A}}\right)^{-1}
$$

holds for all times [13]. Therefore, for the initial and final times,

$$
\frac{\theta_{B}}{\theta_{A}} \geq\left(\frac{y_{B}}{y_{A}}\right)^{-1} \text { or, equivalenty, }\left(\frac{\theta_{B}}{\theta_{A}}\right)^{2} \geq \frac{\kappa_{B}}{\kappa_{A}}
$$


must be fulfilled. The equality in equations 15 and $(16)$ corresponds to the quasi-static case (14).

There exists a minimum time to carry out an adiabatic process [13], namely

$$
\tilde{s}_{\mathrm{f}}=\frac{\left(y_{B}-y_{A}\right)^{2}}{2\left(y_{B} \theta_{B}-y_{A} \theta_{A}\right)} .
$$

This minimum time is reached for a protocol in which the variance and the temperature evolve according to

$$
\tilde{y}(s)=y_{A}+\left(y_{B}-y_{A}\right) \frac{s}{\tilde{s}_{\mathrm{f}}}, \quad \tilde{\theta}(s)=\frac{y_{A} \theta_{A}+\left(y_{B} \theta_{B}-y_{A} \theta_{A}\right) \frac{s}{\tilde{s}_{\mathrm{f}}}}{y_{A}+\left(y_{B}-y_{A}\right) \frac{s}{\tilde{s}_{\mathrm{f}}}},
$$

which are valid in the whole interval $\left[0, \tilde{s}_{\mathrm{f}}\right]$. Therefore, both $\tilde{y}(s)$ and $\tilde{\theta}(s)$ are continuous functions of time, including the initial and final times. The stiffness is given by

$$
\tilde{\kappa}(s)=-\left(\frac{d \tilde{y}(s)}{d s}\right)^{-1} \frac{d \tilde{\theta}(s)}{d s}, \quad 0<s<\tilde{s}_{f} .
$$

and $\tilde{\kappa}(s=0)=\kappa_{A}, \tilde{\kappa}\left(s=\tilde{s}_{\mathrm{f}}\right)=\kappa_{B}$.

The discontinuity at the boundaries of $\kappa(s)$ does not break the adiabatic character of the process: there is no instantaneous heat transfer at the initial and/or final times. Since both the variance $y$ and the temperature $\theta$ are continuous at the boundaries, the integration of the differential of heat, as defined in equation (13), between $s=0$ and $s=0^{+}$(or between $s=\tilde{s}_{f}^{-}$and $s=\tilde{s}_{f}$ ) vanishes. On the contrary, there is an instantaneous contribution to the work at both boundaries.

\section{Irreversible Carnot-like heat engine}

The aim of this work is to study a (stochastic) thermodynamic cycle comprising

the following processes: (i) Isothermal expansion starting from $\left(\kappa_{A}, y_{A}, \theta_{A}\right)$ up to $\left(\kappa_{B}, y_{B}, \theta_{B}=\theta_{A}\right)$ in contact with a hot bath at temperature $\theta_{A}$, (ii) adiabatic expansion starting from $\left(\kappa_{B}, y_{B}, \theta_{B}=\theta_{A}\right)$ up to $\left(\kappa_{C}, y_{C}, \theta_{C}\right)$, (iii) isothermal compression starting from $\left(\kappa_{C}, y_{C}, \theta_{C}\right)$ up to $\left(\kappa_{D}, y_{D}, \theta_{D}=\theta_{C}\right)$ in contact with a cold bath at temperature $\theta_{C}$, and (iv) adiabatic compression going from $\left(\kappa_{D}, y_{D}, \theta_{D}=\theta_{C}\right)$ to $\left(\kappa_{A}, y_{A}, \theta_{A}\right)$. We always choose the normalisation constants (units) such that $\left(\kappa_{A}, y_{A}, \theta_{A}\right)=(1,1,1)$.

As a consequence of the above processes being isothermal/adiabatic, we have the following general identities, $\mathcal{W}_{A B}=-\mathcal{Q}_{A B}, \mathcal{W}_{B C}=\mathcal{E}_{C}-\mathcal{E}_{B}=\theta_{C}-\theta_{A}, \mathcal{Q}_{B C}=0$, $\mathcal{W}_{C D}=-\mathcal{Q}_{C D}, \mathcal{W}_{D A}=\mathcal{E}_{A}-\mathcal{E}_{D}=\theta_{A}-\theta_{C}=-\mathcal{W}_{B C}, \mathcal{Q}_{D A}=0$. We focus on a heat engine, that is, a device that extracts heat from the hot bath and performs work, i.e.

$$
\mathcal{Q}_{A B}=-\mathcal{W}_{A B}>0, \quad \mathcal{W}_{A B}+\mathcal{W}_{B C}+\mathcal{W}_{C D}+\mathcal{W}_{D A}=\mathcal{W}_{A B}+\mathcal{W}_{C D}<0 .(20)
$$

The efficiency of such a device is defined by

$$
\eta \equiv \frac{-\left(\mathcal{W}_{A B}+\mathcal{W}_{C D}\right)}{\mathcal{Q}_{\mathcal{A B}}}=1-\frac{\mathcal{W}_{C D}}{\mathcal{Q}_{A B}}<1
$$


Building an irreversible Carnot-like heat engine

Table 1. Operating points of the Carnot engines. Panels (a) and (b) correspond to the reversible and irreversible versions, respectively.

\begin{tabular}{|l|l|l|l|}
\cline { 2 - 4 } \multicolumn{1}{c|}{$(\mathrm{a})$} & $\kappa$ & $y$ & $\theta$ \\
\hline$A$ & 1 & 1 & 1 \\
\hline$B$ & $\chi$ & $\chi^{-1}$ & 1 \\
\hline$C$ & $\nu^{2} \chi$ & $\nu^{-1} \chi^{-1}$ & $\nu$ \\
\hline$D$ & $\nu^{2}$ & $\nu^{-1}$ & $\nu$ \\
\hline
\end{tabular}

\begin{tabular}{|c|l|l|l|}
\cline { 2 - 4 } \multicolumn{1}{l|}{$(\mathrm{b})$} & $\kappa$ & $y$ & $\theta$ \\
\hline$A$ & 1 & 1 & 1 \\
\hline$B$ & $\chi$ & $\chi^{-1}$ & 1 \\
\hline$C$ & $c \nu^{2} \chi$ & $c^{-1} \nu^{-1} \chi^{-1}$ & $\nu$ \\
\hline$D$ & $d \nu^{2}$ & $d^{-1} \nu^{-1}$ & $\nu$ \\
\hline
\end{tabular}

whereas the power that delivers is given by

$$
\mathcal{P} \equiv \frac{-\left(\mathcal{W}_{A B}+\mathcal{W}_{C D}\right)}{s_{A B}+s_{B C}+s_{C D}+s_{D A}},
$$

where $s_{A B}$ is the time employed for going from $A$ to $B$, and so on.

\subsection{Quasi-static case}

First, we concentrate on the quasi-static limit, that is, we consider a Carnot cycle in which the harmonic oscillator is always at equilibrium. In principle, we must give 12 numbers to characterise the four operating points of the cycle $(A, B, C, D)$, but we have the following constraints: (i) due to normalisation, state $A$ is given, $\left(\kappa_{A}, y_{A}, \theta_{A}\right)=(1,1,1)$ (3 constraints), (ii) points $(B, C, D)$ are equilibrium states (3 constraints), (iii) two isothermal relations $A-B$ and $C-D$ (2 constraints), and (iv) two adiabatic relations $B-C$ and $D-A$ ( 2 constraints). So, we need only $12-3-3-2-2=2$ variables to univocally define the quasi-static cycle.

The cycle is thus completely characterised by the temperature ratio $\nu$ and the compression ratio along the first isotherm $\chi$. The values of the state variables $(\kappa, y, \theta)$ at the operating points of the cycle are collected in panel (a) of Table 1. Note that the isotherm condition implies that $y_{B} / y_{A}=\kappa_{A} / \kappa_{B}=\chi^{-1}$, so the parameter $\chi$ certainly gives the compression ratio along the first isotherm. Hereafter, to keep our wording simpler, we call $\chi$ the compression ratio.

The efficiency of a Carnot cycle

$$
\eta_{\mathrm{C}}=1-\frac{\theta_{C}}{\theta_{A}}=1-\nu,
$$

is well known and can be derived for any system without the knowledge of its state equation through entropic considerations [1]. Here, it can also be explicitly checked by calculating work and heat over the branches of the cycle. The power delivered by this engine is zero, because the processes are quasi-static and thus involve an infinite time.

\subsection{Irreversible Carnot-like cycle at finite speed}

Now we consider a similar cycle, being the only difference that the processes are carried out in a finite time and are thus irreversible. The adiabaticity of the second and third 

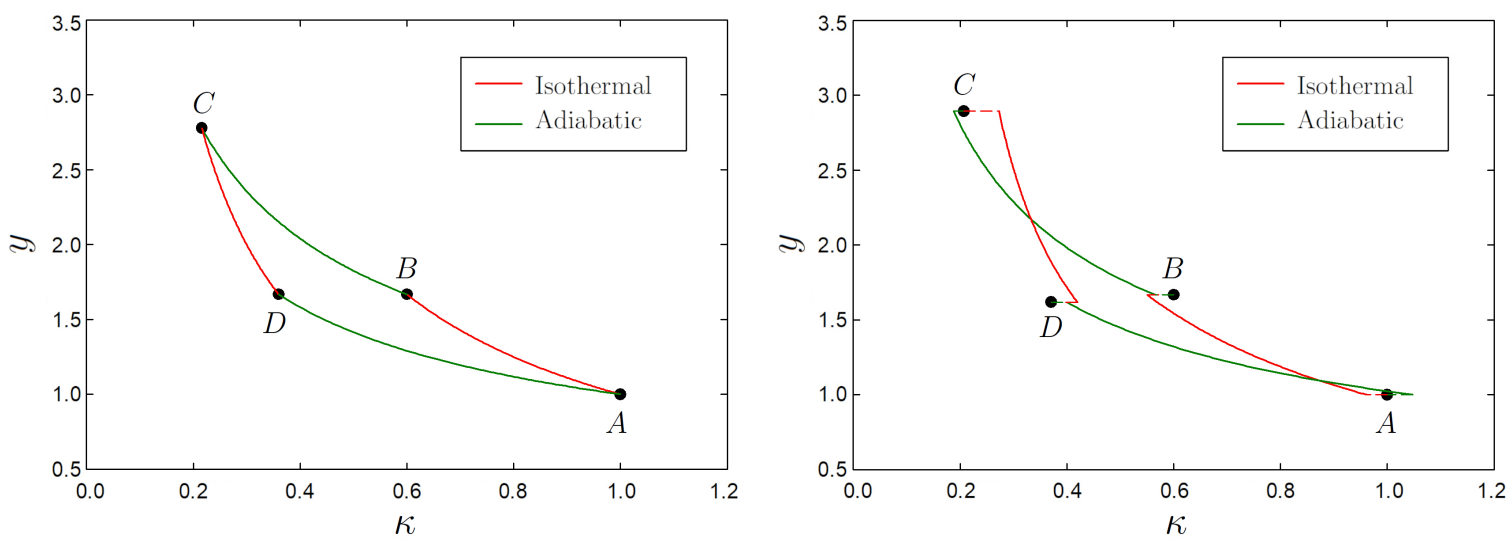

Figure 1. (a) Projection of the movement of the state-point onto the $(\kappa, y)$ plane for a reversible Carnot engine. (b) Projection of the movement of the state-point onto the $(\kappa, y)$ plane for an irreversible Carnot-like engine. Specifically, we have used the parameter values $\nu=0.6, \chi=0.6, c=0.96, d=1.03$ and the corresponding optimal protocols discussed in the text. In both plots, red lines correspond to the isothermal processes and green lines to the adiabatic ones. The dashed segments mark the jumps in the stiffness at the initial and final points of each of the four branches of the cycle.

process impose two inequalities, as expressed by equation (16). Therefore, we have that

$$
\frac{\theta_{C}^{2}}{\theta_{B}^{2}} \geq \frac{\kappa_{C}}{\kappa_{B}}, \quad \frac{\theta_{A}^{2}}{\theta_{D}^{2}} \geq \frac{\kappa_{A}}{\kappa_{D}},
$$

which become equalities only for reversible processes, as those in the previous section. Thus, we need two additional parameters to define the cycle unambiguously, specifically we choose to introduce

$$
c=\kappa_{C} \nu^{-2} \chi^{-1} \leq 1, \quad d=\kappa_{D} \nu^{-2} \geq 1,
$$

which assure that equation (24) is fulfilled. In panel (b) of Table 1, we summarise the values of the state variables $(\kappa, y, \theta)$ at the operating points of this non-equilibrium cycle. A comparative plot of the reversible and irreversible Carnot engines is shown in figure 1 .

In the following, we focus our attention on the maximisation of the power delivered by the engine. Therefore, we build the heat engine that operates at maximum power for fixed operating points $(A, B, C, D)$ or, equivalently, for given values of $(\nu, \chi, c, d)$. We approach the problem of the maximisation of the power defined in equation 22 in a stepwise manner. As discussed in detail below, the main idea is that the global maximum of $\mathcal{P}$ can be obtained as the maximum of maximums, that is, we start by maximising with respect to some parameters keeping the remainder fixed. Afterwards, this maximum can be in turn be maximised with respect to the previously fixed parameters. For instance, maximisation can be performed for a given set $(\nu, \chi, c$ and $d)$, or in a more global fashion, specifying $\nu$ only.

Maximising equation (22) implies to take the shortest possible adiabatic protocols and the minimal work for the isothermal processes. This is readily understood as follows. 
The only dependence on the adiabatic protocols come from $s_{B C}$ and $s_{D A}$, so they have to be minimum in order to give the maximum value for $\mathcal{P}$. With respect to the isothermal processes, for fixed values of $s_{A B}$ and $s_{C D}$, we have to maximise the respective work values $-\mathcal{W}_{A B}$ and $-\mathcal{W}_{C D}$, that is, minimise $\mathcal{W}_{A B}$ and $\mathcal{W}_{C D}$. Therefore, we end up with the optimal processes, either isothermal or adiabatic, discussed in section 3. Making use of equations (10)-(11) for the isothermal processes and equations (17)-(19) for the adiabatic ones, we get

$$
\begin{aligned}
& \mathcal{W}_{A B}=\frac{1}{2} \ln \chi+\frac{1}{s_{A B}}\left(\frac{1}{\sqrt{\chi}}-1\right)^{2}, \quad \mathcal{Q}_{A B}=-\mathcal{W}_{A B}, \\
& \mathcal{W}_{C D}=-\frac{\nu}{2} \ln \frac{c \chi}{d}+\frac{1}{\nu s_{C D}}\left(\frac{1}{\sqrt{c \chi}}-\frac{1}{\sqrt{d}}\right)^{2}, \quad \mathcal{Q}_{C D}=-\mathcal{W}_{C D}, \\
& \tilde{s}_{B C}=\frac{(1-c \nu)^{2}}{2 c \chi \nu^{2}(1-c)}, \quad \tilde{s}_{D A}=\frac{(d \nu-1)^{2}}{2 d \nu^{2}(d-1)}
\end{aligned}
$$

It is worth commenting some points before proceeding further. On the one hand, $\mathcal{W}_{C D}$ is always positive and thus $\mathcal{Q}_{C D}$ is negative; isothermal compression work has to be done on the system and heat is always transferred from the device to the cold bath. On the other hand, in the isothermal expansion, $\mathcal{W}_{A B}$ is negative for large enough $s_{A B}$, but $\mathcal{W}_{A B}$ becomes positive if we intend to compress the system too fast: we have to exert work on the system in that case and moreover $Q_{A B}$ becomes negative and heat is transferred from the system to the hot bath. Therefore, we are not interested here in these too fast isothermal expansions because we would not be building a heat engine in that case. Below we show that this poses no problem because (i) the optimal value $\tilde{s}_{A B}$ yields a negative value of $\mathcal{W}_{A B}$ and (ii) the optimal value $\tilde{s}_{C D}$ makes that $\mathcal{W}_{C D}<-\mathcal{W}_{A B}$, that is, $\mathcal{W}_{C D}+\mathcal{W}_{A B}<0$. Thus, the heat engine conditions are met.

Let us build on the ideas above. We must impose the inequalities 20 to have a heat engine. In particular, these inequalities should hold when $s_{A B}$ and $s_{C D}$ go to infinity (reversible isotherms). It is useful to introduce the definitions

$$
\mathcal{W}_{1} \equiv \lim _{s_{A B} \rightarrow \infty} \mathcal{W}_{A B}=\frac{1}{2} \ln \chi<0, \quad \mathcal{W}_{2} \equiv \lim _{s_{C D} \rightarrow \infty} \mathcal{W}_{C D}=-\frac{\nu}{2} \ln \frac{c \chi}{d}>0,
$$

where we have taken into account that $\chi<1, c \leq 1, d \geq 1$, and

$$
\mathcal{W}_{\infty} \equiv \mathcal{W}_{1}+\mathcal{W}_{2}=\frac{1}{2} \ln \chi-\frac{\nu}{2} \ln \frac{c \chi}{d}<0 .
$$

Although $\mathcal{W}_{1}$ coincides with the value of the work over the first isotherm in the fully reversible engine, neither $\mathcal{W}_{2}$ nor $\mathcal{W}_{\infty}$ does because they depend on $c$ and $d$. The negativeness of $\mathcal{W}_{\infty}$ leads to the constraint

$$
\frac{c}{d}>\chi^{\frac{1-\nu}{\nu}}
$$

Strictly speaking, this constraint has been shown to hold only in the limit as $s_{A B}, s_{C D} \rightarrow$ $\infty$, but below we prove that it also holds for finite-time operation.

Therefore, we just have to maximise the power

$$
\mathcal{P}=\frac{\frac{\nu-1}{2} \ln \chi+\frac{\nu}{2} \ln \frac{c}{d}-\frac{1}{s_{A B}}\left(\frac{1}{\sqrt{\chi}}-1\right)^{2}-\frac{1}{\nu s_{C D}}\left(\frac{1}{\sqrt{d}}-\frac{1}{\sqrt{c \chi}}\right)^{2}}{s_{A B}+\tilde{s}_{B C}+s_{C D}+\tilde{s}_{D A}},
$$


with respect to $s_{A B}$ and $s_{C D}$, by imposing that the partial derivatives of $\mathcal{P}$ with respect to $s_{A B}$ and $s_{C D}$ vanish for the optimal durations of the isothermal processes. In order to write the expressions for $\tilde{s}_{A B}$ and $\tilde{s}_{C D}$, it is convenient to introduce the parameters

$$
\begin{aligned}
& \Delta_{1}=\sqrt{y_{B}}-\sqrt{y_{A}}=\frac{1}{\sqrt{\chi}}-1>0, \\
& \Delta_{2}=\sqrt{y_{D}}-\sqrt{y_{C}}=\frac{1}{\sqrt{\nu}}\left(\frac{1}{\sqrt{d}}-\frac{1}{\sqrt{c \chi}}\right)<0,
\end{aligned}
$$

which measure the expansion and compression of the system in the first and second isotherms, respectively, and

$$
\sigma=\sqrt{1+\frac{\left(\tilde{s}_{B C}+\tilde{s}_{D A}\right)\left(-\mathcal{W}_{\infty}\right)}{\left(\Delta_{1}-\Delta_{2}\right)^{2}}}>1
$$

As a function of these parameters, we can write now that

$$
\tilde{s}_{A B}=\frac{\Delta_{1}\left(\Delta_{1}-\Delta_{2}\right)(1+\sigma)}{-\mathcal{W}_{\infty}}, \quad \tilde{s}_{C D}=\frac{-\Delta_{2}\left(\Delta_{1}-\Delta_{2}\right)(1+\sigma)}{-\mathcal{W}_{\infty}} .
$$

The condition $\mathcal{W}_{\infty}<0$ ensures the positivity of the optimal times.

Using the above definitions, we can write the work values for the optimal durations of the isothermal processes as

$$
\begin{aligned}
& \widetilde{\mathcal{W}}_{A B}=\mathcal{W}_{1}+\frac{\Delta_{1}^{2}}{\tilde{s}_{A B}}=\frac{-\mathcal{W}_{1} \Delta_{2}-\mathcal{W}_{2} \Delta_{1}+\mathcal{W}_{1} \sigma\left(\Delta_{1}-\Delta_{2}\right)}{\left(\Delta_{1}-\Delta_{2}\right)(1+\sigma)}<0, \\
& \widetilde{\mathcal{W}}_{C D}=\mathcal{W}_{2}+\frac{\Delta_{2}^{2}}{\tilde{s}_{C D}}=\frac{\mathcal{W}_{1} \Delta_{2}+\mathcal{W}_{2} \Delta_{1}+\mathcal{W}_{2} \sigma\left(\Delta_{1}-\Delta_{2}\right)}{\left(\Delta_{1}-\Delta_{2}\right)(1+\sigma)}>0 .
\end{aligned}
$$

By combining the expressions above, the total work in the cycle with the optimal durations is found to be

$$
\widetilde{\mathcal{W}}_{A B}+\widetilde{\mathcal{W}}_{C D}=\mathcal{W}_{\infty}-\frac{1}{1+\sigma} \mathcal{W}_{\infty}=\frac{\sigma}{1+\sigma} W_{\infty}<0
$$

The signs of $\widetilde{\mathcal{W}}_{A B}$ and $\widetilde{\mathcal{W}}_{A B}+\widetilde{\mathcal{W}}_{C D}$ show that we have, in fact, a "good" engine. Moreover, we get a physical interpretation for the parameter $\sigma$ : it measures the deviation of the total irreversible work from the value for infinitely slow isothermal processes $\mathcal{W}_{\infty}$. In the limit as $\sigma \rightarrow \infty$, we have that $\widetilde{\mathcal{W}}_{A B}+\widetilde{\mathcal{W}}_{C D} \rightarrow \mathcal{W}_{\infty}$

We have found the optimal values of the times for the isothermal and adiabatic protocols, for given values of the parameters $(\nu, \chi, c, d)$ that univocally define the operating points of our irreversible Carnot-like heat engine. As a function of these parameters, the optimal power is thus given by

$$
\widetilde{\mathcal{P}}=\frac{-\mathcal{W}_{\infty}-\frac{\Delta_{1}^{2}}{\tilde{s}_{A B}}-\frac{\Delta_{2}^{2}}{\tilde{s}_{C D}}}{\tilde{s}_{A B}+\tilde{s}_{B C}+\tilde{s}_{C D}+\tilde{s}_{D A}}=\frac{-\mathcal{W}_{\infty} \frac{\sigma}{1+\sigma}}{\tilde{s}_{A B}+\tilde{s}_{B C}+\tilde{s}_{C D}+\tilde{s}_{D A}},
$$

Later, we address the issue of optimising the cycle further, by looking for the maximum of the $\widetilde{\mathcal{P}}$ as a function of $c, d$ and $\chi$ for a fixed value of the temperature ratio $\nu$.

I Note that, since they do not depend on $s_{A B}$ and $s_{C D}$, we have not substituted explicitly the values of $\tilde{s}_{B C}$ and $\tilde{s}_{D A}$, given by equation $(28)$, so as not to clutter the expression. 


\section{Efficiency at maximum power and the Curzon-Ahlborn bound}

\subsection{Maximal power at fixed temperature and compression ratios}

Let us look into the the efficiency of the maximum power cycle,

$$
\tilde{\eta}=-\frac{\widetilde{\mathcal{W}}_{A B}+\widetilde{\mathcal{W}}_{C D}}{\widetilde{\mathcal{Q}}_{A B}}=1+\frac{\widetilde{\mathcal{W}}_{C D}}{\widetilde{\mathcal{W}}_{A B}}
$$

which depends on $(\nu, \chi, c, d)$. To keep our notation simple, either for $\widetilde{\mathcal{P}}$ in equation 40 or $\tilde{\eta}$ in equation (41), we do not write explicitly the parameters which they depend on. This choice also applies to the remainder of the paper.

Making use of equation (37), we can rewrite $\tilde{\eta}$ as

$$
\tilde{\eta}=\underbrace{1-\nu}_{\eta_{\mathrm{C}}}+\frac{(\nu-1)\left(\mathcal{W}_{1} \Delta_{2}+\mathcal{W}_{2} \Delta_{1}\right)-\left(\mathcal{W}_{2}+\mathcal{W}_{1} \nu\right) \sigma\left(\Delta_{1}-\Delta_{2}\right)}{\mathcal{W}_{1} \Delta_{2}+\mathcal{W}_{2} \Delta_{1}-\mathcal{W}_{1} \sigma\left(\Delta_{1}-\Delta_{2}\right)}
$$

All the terms in the denominator are clearly positive, whereas all the terms in the numerator are negative by taking into account that $\mathcal{W}_{2}+\mathcal{W}_{1} \nu=-(\nu / 2) \ln (c / d)>0$. Therefore, $\tilde{\eta}<\eta_{\mathrm{C}}$ : the efficiency is always below the Carnot bound, as expected.

On the other hand, the comparison with the Curzon-Ahlborn bound 14, 20, 41, 42,

$$
\eta_{\mathrm{CA}}=1-\sqrt{\nu}
$$

requires a more detailed analysis. Let us investigate two different cases. First, we consider values of the ratio $c / d$ such that $\chi^{-1+\nu^{-1}}<c / d<\chi^{-1+\nu^{-1 / 2}}$, which entails that $\mathcal{W}_{2}+\sqrt{\nu} \mathcal{W}_{1}>0$. for which

$$
\underbrace{(\sqrt{\nu}-1)}_{<0}(\underbrace{\mathcal{W}_{1} \Delta_{2}}_{>0}+\underbrace{\mathcal{W}_{2} \Delta_{1}}_{>0})-\underbrace{\left(\mathcal{W}_{2}+\mathcal{W}_{1} \sqrt{\nu}\right)}_{>0} \sigma \underbrace{\left(\Delta_{1}-\Delta_{2}\right)}_{>0}<0 .
$$

In this region, a manipulation similar to the one done for showing that $\eta<\eta_{\mathrm{C}}$ gives

$$
\tilde{\eta}=1-\sqrt{\nu}+\frac{(\sqrt{\nu}-1)\left(\mathcal{W}_{1} \Delta_{2}+\mathcal{W}_{2} \Delta_{1}\right)-\left(\mathcal{W}_{2}+\mathcal{W}_{1} \sqrt{\nu}\right) \sigma\left(\Delta_{1}-\Delta_{2}\right)}{\mathcal{W}_{1} \Delta_{2}+\mathcal{W}_{2} \Delta_{1}-\mathcal{W}_{1} \sigma\left(\Delta_{1}-\Delta_{2}\right)} .
$$

Again, the denominator and the numerator are positive and negative respectively, which leads to the inequality $\tilde{\eta}<\eta_{C A}$. Nevertheless, for the complementary case, $\chi^{-1+\nu^{-1 / 2}}<c / d<1$, we can no longer assure that the Curzon-Ahlborn is an upper bound. Indeed, in the double limit as $(c, d) \rightarrow(1,1)$, we have that $\tilde{s}_{B C}$ and $\tilde{s}_{D A}$ diverge for fixed $\nu<1$. In that limit, not only do the adiabatic processes become quasi-static but also the isothermal ones, recovering the quasi-static Carnot engine introduced in section 4.1, with optimal efficiency $\lim _{(c, d) \rightarrow(1,1)} \tilde{\eta}=\eta_{\mathrm{C}}$. Because of continuity, we can always find values of $c$ and $d$, given a value of $\chi$, such that the efficiency of our optimal heat engine is arbitrarily close to the Carnot value and thus greater than the CurzonAhlborn bound. However, it has to be taken into account that the optimal power for this case is very small, because the denominator in equation 40 diverges. In section 1 of the Supplementary Material, we consider the leading order of $\tilde{\eta}$ and $\tilde{\mathcal{P}}$.

To illustrate the above results, we present in figure 2 a density plot of the optimal power, equation (40), and the corresponding efficiency, equation (41), as a function of $c$ 

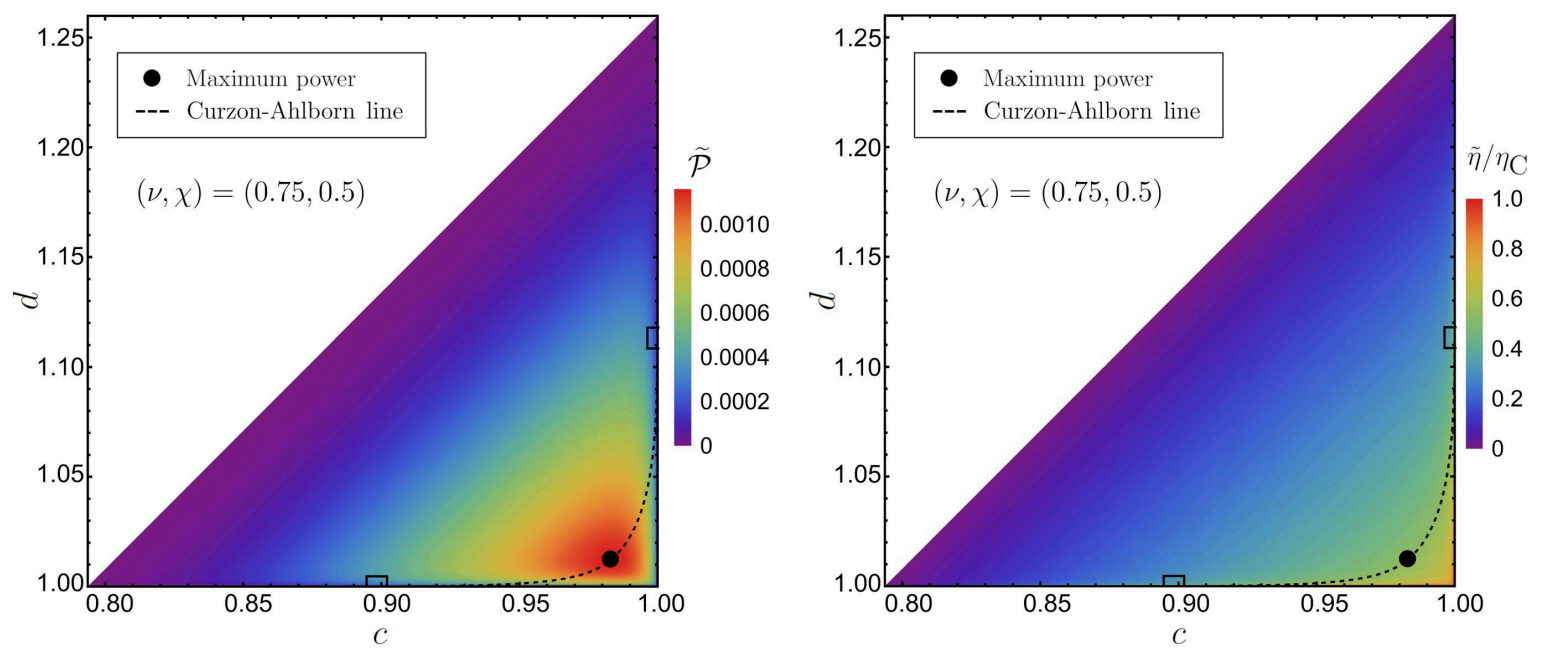

Figure 2. Density plots of the optimal power (left) and its corresponding efficiency (right) in the $(c, d)$ plane. The curves where $\tilde{\eta}=\eta_{\mathrm{CA}}$ (dashed line), with its initial and final points (open squares) over the axes $d=1$ and $c=1$, respectively, and the point at which the maximum power (circle) is reached, are displayed in both panels. We have taken $\nu=0.75$ and $\chi=0.5$.

and $d$. Specifically, we consider given values of the temperature ratio $\nu=0.75$ and the compression ratio $\chi=0.5$. The constraint (31) entails that the meaningful region in the plane $(c, d)$ is a right triangle of vertices $\left(c_{\min }=\chi^{\frac{1-\nu}{\nu}}, 1\right),(1,1)$ and $\left(1, d_{\max }=c_{\min }^{-1}\right)$. Within this region, we can define another right triangle with the right angle in the same vertex and the hypotenuse given by the line $d=\chi^{\frac{\sqrt{\nu}-1}{\sqrt{\nu}}} c$, above which we know that $\tilde{\eta}<\eta_{\mathrm{CA}}$. Below the aforementioned line, we cannot assure that $\tilde{\eta}<\eta_{\mathrm{CA}}$ and in the limit as $(c, d) \rightarrow(1,1)$ we know that $\tilde{\eta} \rightarrow \eta_{\mathrm{C}}$. The curve over which $\tilde{\eta}=\eta_{\mathrm{CA}}$, which departs from the hypotenuse vertices (open squares) of this second triangle and is fully contained within it, has been evaluated numerically and plotted (dashed line) along with the point of delivery of maximum power (circle).

There are several implications that can be drawn from this analysis. First, along all the sides of the delimiting triangle, the maximum power is zero because some of the optimal times diverge. Second, as a consequence of the previous point and the positiveness of $\widetilde{\mathcal{P}}$, there always appears a maximum of the optimal power as a function of $(c, d)$ (for fixed $\nu$ and $\chi$ ), at a certain point $\tilde{c}, \tilde{d}$. Third, the numerical estimate for this maximum is very close to the dotted line, at which $\tilde{\eta}=\eta_{C A}$. This last observation is especially robust for either small $\chi$ or large $\nu$, as can be seen in section 2 of the Supplementary Material, in which analogous plots for different couples of values $(\nu, \chi)$ are presented. 


\subsection{Maximal power for fixed temperature ratio $\nu$}

The numerical analysis shown in figure 2 suggests that studying further the maximum power that can be achieved for fixed values of $\nu$ and $\chi$, that is, as a function of $c$ and $d$, may be illuminating. This is a meaningful physical question: recall that the reversible Carnot engine is completely determined by these two parameters. Moreover, its efficiency $\eta_{\mathrm{C}}$ does not depend on the compression ratio $\chi$, which makes interesting even a further maximisation in the compression ratio $\chi$.

It is possible to address this problem by maximising again the optimal power in equation (40) with respect to $c$ and $d$, and finally with respect to $\chi$. Doing so analytically is not feasible since it involves transcendental equations. Nevertheless, a systematic asymptotic analysis can be carried out for $\nu \rightarrow 1$. In this regime, the main idea is to expand all the physical quantities in powers of $\eta_{\mathrm{C}}=1-\nu$. In order to avoid cluttering the information flow with the technicalities of the asymptotic analysis, we present the detailed calculation in sections 3 and 4 of the Supplementary Material. Therein, it is shown that the expansions of $\widetilde{\mathcal{P}}$ and $\tilde{\eta}$ in the Carnot efficiency up to order $\eta_{\mathrm{C}}^{4}$ and $\eta_{\mathrm{C}}^{3}$, respectively, are

$$
\begin{aligned}
& \tilde{\mathcal{P}}=\frac{\eta_{\mathrm{C}}^{2}}{16}-\frac{\eta_{\mathrm{C}}^{5 / 2}}{8}+\frac{5}{48} \eta_{\mathrm{C}}^{3}-\frac{11}{144} \eta_{\mathrm{C}}^{7 / 2}+\frac{937}{17280} \eta_{\mathrm{C}}^{4}+O\left(\eta_{\mathrm{C}}^{9 / 2}\right), \\
& \tilde{\eta}=\frac{\eta_{\mathrm{C}}}{2}+\frac{\eta_{\mathrm{C}}^{2}}{8}+\frac{\eta_{\mathrm{C}}^{3}}{32}+O\left(\eta_{\mathrm{C}}^{7 / 2}\right) .
\end{aligned}
$$

We recall that the expansion of the Curzon-Ahlborn efficiency is

$$
\eta_{\mathrm{CA}}=\frac{\eta_{\mathrm{C}}}{2}+\frac{\eta_{\mathrm{C}}^{2}}{8}+\frac{\eta_{\mathrm{C}}^{3}}{16}+O\left(\eta_{\mathrm{C}}^{4}\right)
$$

Similarly to the situation reported in Refs. [14, 30 the first two terms in the expansion of $\tilde{\eta}$ in powers of $\eta_{\mathrm{C}}$ coincide with those in $\eta_{\mathrm{CA}}$ and the deviation occurs in the third term, of the order of $O\left(\eta_{\mathrm{C}}^{3}\right)$. The obtained efficiency at maximum power is smaller than the Curzon-Ahlborn bound, similarly to the situation found in Ref. 14.

In figure 3, we plot the efficiency at maximum power as a function of $\nu$. Power has been numerically maximised over $c, d$ and $\chi$. The obtained efficiency $\tilde{\eta}$ is compared with (i) the Curzon-Ahlborn bound, (ii) the efficiency for the engine with instantaneous "adiabatic" branches developed in $\left[14, \eta_{\text {ref }}^{(I)}=2 \eta_{\mathrm{C}} /\left(4-\eta_{\mathrm{C}}\right)\right.$, and (iii) the efficiency obtained for large dissipation in the recent proposal, using a fast forward approach [18, to build a Carnot-like engine, $\eta_{\mathrm{ref}}^{(I I)}=(1-\nu)(1+\sqrt{\nu}) /[2+\sqrt{\nu}(1+\nu)] \leq \eta_{\mathrm{ref}}^{(I)}$. It is clearly observed that $\tilde{\eta} \geq \eta_{\text {ref }}^{(I)}$ for all $\nu$, with the difference between them increasing as $\nu$ decreases. Moreover, the closeness between the efficiency of our engine at maximum power and the Curzon-Ahlborn bound goes beyond our expectations based on the asymptotic analysis, holding not only within the limit $\nu \rightarrow 1$ but also for the whole range of $\nu$. Specifically, the relative deviation between our numerical values for efficiency at maximum power and the Curzon-Ahlborn bound always remains under $2 \%$. Therefore,

+ See equations (24) and (25) in that paper. However, the reverse situation has also been found, see for instance equation (20) in Ref. 30]. 


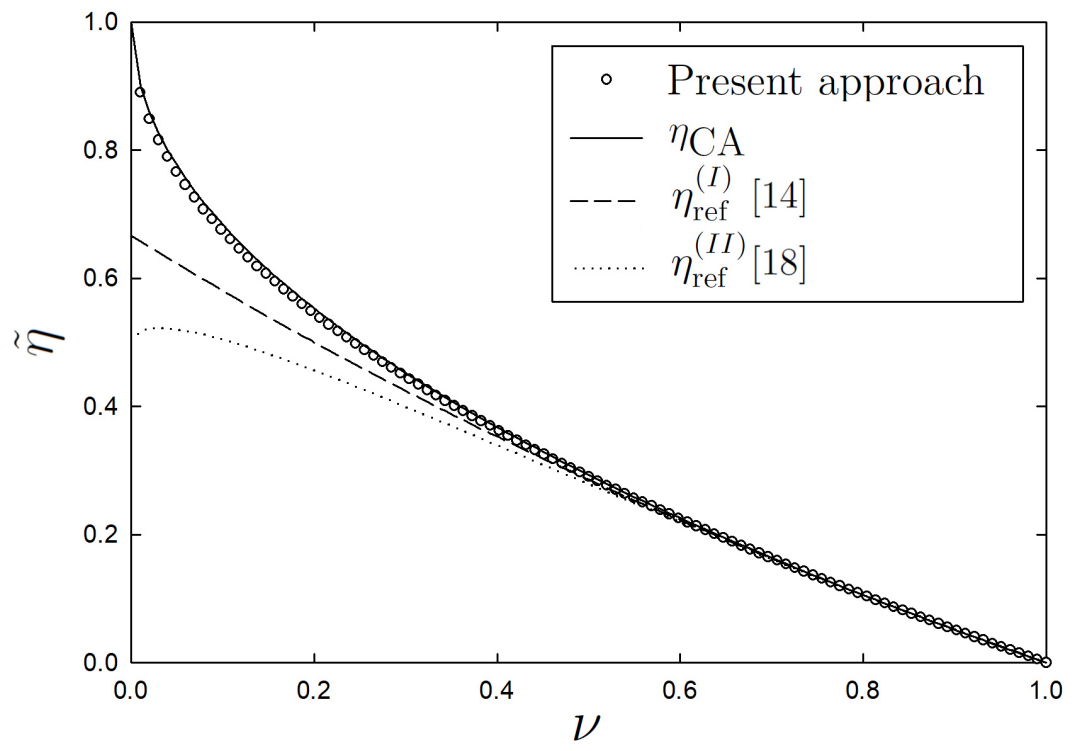

Figure 3. Efficiency at maximum power as a function of the temperature ratio $\nu$. The value obtained for the efficiency, once that the optimisation of the power is numerically performed for the rest of parameters, is almost indistinguishable from the Curzon-Ahlborn bound $\eta_{\mathrm{CA}}$. Our construction develops a better efficiency compared with those shown in Refs. 14] and 18.

our novel irreversible Carnot-like heat engine is certainly a very efficient one at maximum power.

\section{Conclusions}

In this work, we have put forward an irreversible Carnot-like heat engine. Our model system is a Brownian particle trapped in a harmonic potential, in the overdamped regime. The adiabatic branches of the proposed cycle are truly adiabatic in the classical thermodynamic sense: at every point thereof, there is no heat exchange with the thermal bath. Of course, the heat exchange vanishes in average: it is impossible to completely decouple the colloidal particle from the surrounding fluid. Therefore, our locally adiabatic branches contrast with the approach followed in other works, in which the system has a non-vanishing heat exchange in the "adiabatic" parts of the cycle [4, 14, 17, 19].

The cycle of the reversible Carnot heat engine is completely characterised by the temperature ratio $\nu$ and the compression ratio $\chi$. For our irreversible counterpart of the Carnot heat engine, we need two more parameters in order to fully characterise the four operating points of the cycle: the adiabatic condition imposes restrictions on-but does not univocally define - the operating points.

We have thoroughly studied the performance of the Carnot-like heat engine at maximum power. We have adopted a step-by-step optimisation approach. First, the 
maximum power is shown to be obtained for the optimal protocols for both isothermalmaximum work [14, 31, 32 -and adiabatic - minimum duration [13]-branches. In a second step, we have optimised the power over the duration of the isothermal processes. These two stages of the optimisation have been carried out for fixed operation points in the state space $(\kappa, y, T)$ - (stiffness, variance of position, temperature). Finally, we have maximised the power over the operation points by just fixing the temperature ratio $\nu$.

The efficiency at maximum power for our heat engine is very close to the CurzonAhlborn bound. This behaviour is predicted by an asymptotic analysis for $\nu \rightarrow 1$. Nevertheless, we have numerically shown that this result remarkably holds for the whole range of temperature ratios, well beyond the asymptotic prediction. This implies that our cycle is a close to optimal choice for building an efficient mesoscopic heat engine, as compared with the theoretical predictions for other constructions 14,18]. Possible venue for future work includes the study of fluctuations, beyond the mean scenario reported here 43,44 .

\section{Acknowledgment}

A.P. acknowledges financial support from the Spanish Agencia Estatal de Investigación through Grant No. PGC2018-093998-B-I00, partially financed by the European Regional

Development Fund. C.A.P. acknowledges the support from University of Padova through Project No. STARS-Stg (CdA Rep. 40, 23.02.2018) BioReACT grant. This work has also been financially supported by the Agence Nationale de la Recherche through Grant No. ANR-18-CE30-0013 (D.G.-O., E.T.).

\section{References}

[1] Callen H 1985 Thermodynamics and an Introduction to Thermostatistics (Wiley)

[2] Sekimoto K 2010 Stochastic Energetics (Springer)

[3] Seifert U 2012 Reports on Progress in Physics 75126001

[4] Ciliberto S 2017 Physical Review X 7021051

[5] Martínez I A, Petrosyan A, Guéry-Odelin D, Trizac E and Ciliberto S 2016 Nature Physics 12 $843-846$

[6] Muratore-Ginanneschi P and Schwieger K 2017 Entropy 19379

[7] Li G, Quan H T and Tu Z C 2017 Physical Review E 96012144

[8] Chupeau M, Ciliberto S, Guéry-Odelin D and Trizac E 2018 New Journal of Physics 20075003

[9] Chupeau M, Besga B, Guéry-Odelin D, Trizac E, Petrosyan A and Ciliberto S 2018 Physical Review E 98010104

[10] Albay J A C, Wulaningrum S R, Kwon C, Lai P Y and Jun Y 2019 Physical Review Research 1 033122

[11] Albay J A C, Lai P Y and Jun Y 2020 Applied Physics Letters 116103706

[12] Guéry-Odelin D, Ruschhaupt A, Kiely A, Torrontegui E, Martínez-Garaot S and Muga J 2019 Reviews of Modern Physics 91045001

[13] Plata C A, Guéry-Odelin D, Trizac E and Prados A 2020 Physical Review E 101032129

[14] Schmiedl T and Seifert U 2008 EPL (Europhysics Letters) 8120003

[15] Bo S and Celani A 2013 Physical Review E 87 050102(R)

[16] Tu Z C 2014 Physical Review E 89052148 
[17] Martínez I A, Roldán E, Dinis L, Petrov D, Parrondo J M R and Rica R A 2016 Nature Physics $1267-70$

[18] Nakamura K, Matrasulov J and Izumida Y 2020 arXiv:2003.05099 [cond-mat]

[19] Martínez I A, Roldán E, Dinis L, Petrov D and Rica R A 2015 Physical Review Letters 114120601

[20] Curzon F L and Ahlborn B 1975 American Journal of Physics 43 22-24

[21] Andresen B, Salamon P and Berry R S 1977 The Journal of Chemical Physics 66 1571-1577

[22] De Vos A 1985 American Journal of Physics 53 570-573

[23] Hoffmann K H, Watowich S J and Berry R S 1985 Journal of Applied Physics 58 2125-2134

[24] Chen L and Yan Z 1989 The Journal of Chemical Physics 90 3740-3743

[25] Chen J 1994 Journal of Physics D: Applied Physics 27 1144-1149

[26] Van den Broeck C 2005 Physical Review Letters 95190602

[27] Esposito M, Lindenberg K and Van den Broeck C 2009 Physical Review Letters 102130602

[28] Sheng S and Tu Z C 2015 Physical Review E 91022136

[29] Tu Z C 2008 Journal of Physics A: Mathematical and Theoretical 41312003

[30] Esposito M, Lindenberg K and Broeck C V d 2009 EPL (Europhysics Letters) 8560010

[31] Schmiedl T and Seifert U 2007 Physical Review Letters 98108301

[32] Plata C A, Guéry-Odelin D, Trizac E and Prados A 2019 Physical Review E 99012140

[33] Martínez I A, Roldán E, Parrondo J M R and Petrov D 2013 Physical Review E 87(3) 032159

[34] Pontryagin L S 1987 Mathematical Theory of Optimal Processes (CRC Press)

[35] Liberzon D 2012 Calculus of Variations and Optimal Control Theory: A Concise Introduction (Princeton University Press)

[36] Band Y B, Kafri O and Salamon P 1982 Journal of Applied Physics 53 8-28

[37] Aurell E, Mejía-Monasterio C and Muratore-Ginanneschi P 2011 Physical Review Letters 106 250601

[38] Aurell E, Mejía-Monasterio C and Muratore-Ginanneschi P 2012 Physical Review E 85020103

[39] Newman E and Bergmann P G 1955 Physical Review 99 587-592

[40] Tolle H 2012 Optimization Methods (Springer Science \& Business Media)

[41] Esposito M, Kawai R, Lindenberg K and Van den Broeck C 2010 Physical Review Letters 105 150603

[42] Apertet Y, Ouerdane H, Goupil C and Lecoeur P 2017 Physical Review E 96022119

[43] Gingrich T R, Rotskoff G M, Vaikuntanathan S and Geissler P L 2014 New Journal of Physics 16 102003

[44] Polettini M, Verley G and Esposito M 2015 Physical Review Letters 114050601 